\title{
Financiamiento de redes integradas de servicios de salud
}

\section{Camilo Cid Pedraza ${ }^{1}$}

Forma de citar Cid Pedraza C. Financiamiento de redes integradas de servicios de salud. Rev Panam Salud Publica. 2020;44:e121. https:// doi.org/10.26633/RPSP.2020.121

Resumen

Los países de América Latina y el Caribe necesitan aumentar sus recursos públicos en salud para ampliar el acceso equitativo y eficiente a la salud. El aumento debe financiar un modelo específico que ha mostrado evidencia de eficacia, como el de redes integradas de servicios de salud (RISS) basadas en atención primaria de salud. La literatura global no ha prestado suficiente atención al financiamiento a las RISS; más bien se ha focalizado en establecimientos y agentes aislados, así como en mecanismos específicos. Sin embargo, en la Región de las Américas su desarrollo es una necesidad desde hace años. Una RISS es un conjunto de organizaciones de salud que ofrece intervenciones y servicios de salud coordinados a una población bajo su cargo y asume la responsabilidad de salud y económica en el logro de mejores resultados de salud. Un sistema de pago a una RISS debe ir dirigido a promover la integralidad de la atención y a fomentar un enfoque centrado en el ciclo de vida de las personas, la articulación y la coordinación de servicios. El presupuesto poblacional ajustado por riesgos se muestra como un mecanismo posible y potente para apoyar el logro de los objetivos. Su desarrollo requiere reconocer que la forma de financiamiento no responderá por sí sola a los desafíos y que se necesita, a la vez, de la planificación y de la gestión sanitarias. Se requiere abordar los desafíos técnicos, políticos e institucionales para tener éxito en este esfuerzo, que a su vez debe estar inserto en el proceso global de transformación de los sistemas de salud hacia la salud universal.

Palabras clave Asignación de recursos para la atención de salud; capitación; sistemas de salud; financiación de la atención de la salud; Américas.

Los países de las Américas, a través de la Estrategia de Salud Universal que promueve la Organización Panamericana de la Salud (OPS/OMS) (1) han acordado que para avanzar hacia la salud universal es necesario aumentar y mejorar el financiamiento con equidad y eficiencia, apuntando a lograr una meta referencial del $6 \%$ del producto interno bruto (PIB) de gasto público en salud, de modo de poder brindar acceso universal a la salud a sus poblaciones con una adecuada protección financiera.

Sin embargo, no se trata solamente de aumentar el gasto en salud; también es necesario promover la eficiencia de los sistemas de salud a fin de garantizar un acceso a la salud equitativo y poner el foco en la organización de servicios de salud con

\footnotetext{
Organización Panamericana de la Salud/Organización Mundial de la Salud, Washington, DC. Estados Unidos de América. $₫$ Camilo Cid Pedraza, cidcam@ paho.org
}

modelos de atención centrados en las personas y las comunidades y la provisión de servicios de calidad. Para ello, los sistemas de pago deben estar alineados con los objetivos del sistema de salud. (1) Una adecuada asignación de los recursos debe estar orientada a aumentar la equidad en el acceso al dirigirse prioritariamente al primer nivel de atención para mejorar su capacidad resolutiva y su capacidad de articulación de las redes integradas de servicios de salud (RISS) en un contexto de desarrollo de la estrategia de atención primaria de salud (APS). Las RISS son un conjunto de organizaciones de salud que prestan servicios de salud integrales a una población definida. $(2,3)$

En este marco, el presente artículo busca aportar ideas acerca de cómo se deberían financiar aquellas RISS que reúnan las características descritas con base en evidencia bibliográfica teórica y de casos implementados en sistemas de salud de diversos países, ya sea a nivel nacional o subnacional, en todo 
el sistema de salud o en parte de él. El artículo, sin embargo, no pretende hacer una revisión de tales casos sino sistematizar algunas recomendaciones para el financiamiento de RISS basadas en evidencia. Se reseñan primero aspectos asociados al desarrollo conceptual en el tema de pagos a proveedores de salud y la notable escasez de tratamiento del concepto de redes integradas. Luego se argumenta que el objeto central de un sistema de financiamiento de proveedores de salud y de la organización de los servicios de atención de salud debieran ser las RISS basadas en APS, y finalmente se abordan algunas ideas orientadoras acerca de cuáles son los mejores sistemas de pago en este contexto.

\section{FINANCIAMIENTO Y SISTEMAS DE PAGO}

En el marco de la reflexión acerca de la asignación de recursos a través de sistemas y mecanismos de pago o de compra estratégica ${ }^{1}$ a proveedores de salud usualmente se considera como objeto del análisis a un agente individual o un grupo de ellos (p. ej., un hospital o un grupo de ellos, un centro de salud del primer nivel de atención o varios de ellos, los médicos o equipos de salud, etc.). Sin embargo, cuando este tipo de políticas se impulsa de manera parcial puede resultar en

aplicaciones poco efectivas, en el mejor de los casos, o en implementaciones francamente inconvenientes, en muchos de ellos, debido a que en ese caso los sistemas de pago pueden descuidar las necesidades de coordinación e integración de la atención, priorizar a un sector de la provisión por sobre los otros, e incluso establecer incentivos contradictorios o directamente nocivos para los objetivos estratégicos de la red en su conjunto. $(4,5)$

Incluso cuando se revisa la sistematización de conocimiento acerca de sistemas de pago desarrollada por organismos internacionales en textos del tipo "manuales" se aprecia una ausencia - O escaso tratamiento- del tema de pagos a organizaciones sanitarias integradas o RISS en tanto objeto de financiamiento. En el Cuadro 1 se presentan manuales recientes sobre sistemas de pago para contrastar esta afirmación. Se analizó la cobertura temática que ofrecen en cuanto distintos modelos de pagos y si consideran, además, articulaciones o coordinaciones entre niveles de atención. De los siete manuales recogidos entre 2005 y 2016, sólo uno de ellos aborda este tópico de manera explícita y sólo aquellos artículos dedicados más a las políticas de salud muestran preocupación por la coordinación. En el conjunto aparecen como prioridad el pago a los hospitales, y en segundo término el pago al primer nivel de atención, inclusive a los médicos de este.

Sin embargo, el tema del financiamiento a las RISS ha sido de interés en la literatura y existen experiencias aplicadas, como la de España o la del Reino Unido $(13,14)$. Esta área también ha despertado interés en la Región de las Américas, inclusive en Estados Unidos en el mundo de las Organizaciones de Cuidados de Salud y en América Latina y el Caribe (ALC) en las

\footnotetext{
${ }^{1}$ Usamos el concepto de sistemas de pago para referirnos a un conjunto de mecanismos que hacen parte de la manera de asignar recursos a los proveedores de un sistema de salud. Un mecanismo de pago, por lo tanto, es cada elemento específico del sistema de pago como, por ejemplo, el pago por caso o el pago per cápita. En este artículo el concepto de compra estratégica da cuenta de una sistematización sobre diseño y aplicación de sistemas de pago, que en cuanto al objetivo del trabajo no presenta mayor diferencia con el concepto de sistemas de pago excepto cuando se indican algunas características deseables de los sistemas de pago hacia el final del artículo.
}

publicaciones regionales de la Organización Panamericana de la Salud/Organización Mundial de la Salud (OPS/OMS) y de algunos países. En 2006, por ejemplo, trabajos provenientes de Chile planteaban el tema del financiamiento a organizaciones sanitarias integradas mediante una cápita ajustada por riesgo (15). En 2010, la OPS abordó la definición de RISS y el pago capitado (2); en 2014 la Estrategia de salud universal de la OPS planteaba la necesidad de alinear los mecanismos de pago a las RISS (1); y en Salud en las Américas 2017 se presentaba dentro de las recomendaciones. (16) Más recientemente, una comisión de alto nivel convocada por la OPS señalaba nuevamente esta necesidad. (17) Existen, asimismo, estudios revisados por pares y literatura gris sobre la experiencia de varios países, como Ecuador (18) y Perú (19).

Es posible, entonces, discutir acerca de cómo avanzar hacia mejores alternativas de financiamiento de las RISS que superen la visión aislada o parcial del tipo de proveedores, los niveles de atención o el tipo de mecanismo de pago, para así realmente alinear los sistemas de financiamiento con los objetivos de salud y hacer una contribución significativa al éxito de la estrategia de RISS basada en la APS.

\section{POR QUÉ REDES INTEGRADAS DE SERVICIOS DE SALUD}

Los servicios de atención de salud involucran a múltiples proveedores que necesitan actuar de manera coordinada. A su vez, la coordinación de los proveedores implica la concertación de las distintas actividades y funciones requeridas para dar atención integral a lo largo de un continuo de atención, de manera que estas se armonicen y se alcance el objetivo deseado -común para el conjunto de proveedores de una Red-, de otorgar intervenciones y atención de salud centradas en las personas y las comunidades. De este modo, la coordinación de servicios de salud está centrada en la interacción entre proveedores, y cuando esa coordinación es suficientemente efectiva ${ }^{2}$ se considera que la atención está integrada. (20)

Para lograr la integración y combatir la fragmentación de los sistemas de salud, que dificulta el acceso a la salud, se promueve la estrategia de atención primaria de salud, cuya expresión operativa son las RISS. (2) Consecuentemente, desde el ámbito económico y de financiamiento de la salud, estas políticas enfatizan la colaboración entre proveedores más que la competencia (21) y promueven las RISS como la forma más eficiente y equitativa de solucionar los problemas de salud de las personas, las familias y las comunidades. Las RISS permiten la organización y coordinación de distintos proveedores que se desempeñan en un territorio específico, tienen distintos niveles de complejidad y ofrecen una atención integral a través de un continuo de prestaciones dirigidas a una población determinada -donde el primer nivel de atención desempeña un rol esencial de articulación-, y que se responsabilizan de sus costos, su calidad y sus resultados de salud. En efecto, una RISS que funciona de manera óptima es una trama de organizaciones de salud que ofrece un conjunto de intervenciones y servicios de salud coordinados a una

\footnotetext{
${ }^{2}$ Somos conscientes de que esta afirmación implica que existen niveles de integración y un mecanismo de evaluación. No es nuestra intención desarrollar tales aspectos, pero ideas e instrumentos relacionados pueden encontrarse en la referencia 2 .
} 
CUADRO 1. Contenidos de siete manuales de política aplicada sobre sistemas de pago a proveedores

\begin{tabular}{|c|c|c|c|c|c|}
\hline \multirow[b]{2}{*}{ Editor } & \multicolumn{5}{|c|}{ Se trata el tema de pagos a: } \\
\hline & $\begin{array}{l}\text { Primer nivel de } \\
\text { atención }\end{array}$ & Hospitales & $\begin{array}{l}\text { Ambulatorio de } \\
\text { Especialidad }\end{array}$ & Médicos & Redes/RISS \\
\hline Observatorio Europeo/OMS, 2005 (6) & + & ++ & + & -- & -- \\
\hline Banco Mundial, 2009 (8) & +++ & +++ & -- & ++ & -- \\
\hline Observatorio Europeo/OMS, 2011 (9) a & -- & +++ & -- & -- & -- \\
\hline OCDE, 2016 (12) & ++ & ++ & + & ++ & ++ \\
\hline
\end{tabular}

Fuente: Elaboración propia

+): implica que se trata el tema en el texto en grado variable (+, ++ y +++ según la intensidad con que se aborda el tema);

a (-): ausencia del tema en el texto. OMS, Organización Mundial de la Salud; RISS, redes integradas de servicios de salud; OCDE, Organización para la Cooperación y el Desarrollo Económicos

población bajo su cargo y asume la responsabilidad de salud y económica en el logro de mejores resultados de salud en la población a la que sirve.

En términos económicos, la integración asistencial supone la integración vertical entre niveles de atención para producir servicios de salud aprovechando las economías de escala, los ajustes a los costos administrativos y de transacción y las posibilidades de adelantarse a la demanda cuando hay coordinación y planificación adecuadas, en especial en el manejo de las enfermedades crónicas. Es decir, la integración permite producir de la manera más eficiente posible la atención de salud que responda a la población de la cual la RISS es responsable y cuyos consumos de salud implican un riesgo financiero.

Sin duda, el tipo de integración que exista en cada caso concreto determina la intensidad con que se pueda transmitir los efectos buscados, ya que muchas veces los sistemas de salud se encuentran fracturados entre niveles de atención por esquemas de descentralización fallidos o que han ido más allá de lo necesario, en cuyo caso predomina la integración virtual, (22) más que la real.

Muchos países se esfuerzan en este camino de integración de sus sistemas de atención de salud, como España, Reino Unido, Italia, Portugal, Finlandia o Australia, entre los países miembros de la Organización para la Cooperación y el Desarrollo Económico (OCDE). En la Región de las Américas, sin duda son destacables los esfuerzos de Canadá, Cuba, Brasil en el Sistema Único de Salud (SUS), Chile y su sector público llamado Sistema Nacional de Servicios de Salud (SNSS), y Costa Rica. En EE.UU., con una realidad en salud muy heterogénea y compleja, existen experiencias en el financiamiento de las Organizaciones para el Cuidado de la Salud (HMO), especialmente en California con Kaiser Permanente ${ }^{3}$. Existen, asimismo, ejemplos más recientes en el marco de la implementación de las Organizaciones Responsables de Cuidados (Accountable Care Organizations, ACO), introducidas en la última reforma a la salud (Patient Protection and Affordable Care Act, coloquialmente llamada Obamacare). $(23,24)$

\footnotetext{
${ }^{3}$ Kaiser Permanente es una Organización para el Cuidado de la salud (HMO) que opera fundamentalmente en el Oeste de EE.UU. (principalmente en California, aunque tiene afiliados también en Washington DC y Maryland). Tiene más de 12 millones de afilados y es una de las organizaciones integradas de salud más grandes del país.
}

\section{FINANCIAMIENTOY ASIGNACIÓN DE RECURSOS PARA EL DESARROLLO DE RISS}

Entre los elementos clave para el éxito de una RISS -que abarcan desde recursos humanos suficientes, adecuados, motivados y que se desempeñan con una visión compartida, hasta contar con poblaciones definidas por las cuales responder-, está el sistema de pagos, que debe promover incentivos alineados con los objetivos de la RISS. Tal como sucede en ausencia de una estrategia y una implantación adecuada del sistema de organización integrada, si faltan los incentivos o estos no están bien diseñados se hace difícil el desempeño adecuado de una RISS. No obstante, estos incentivos son solo un aspecto; el sistema de pagos, a la vez, debe ser compatible con los mecanismos de coordinación y planificación de salud, incluido el presupuesto.

Lo anterior quiere decir que antes del pago y de la asignación de los recursos financieros, existen una planificación sanitaria y decisiones de asignación realizadas en función de diagnósticos epidemiológicos y sociales que han determinado unos objetivos de salud (idealmente, reflejados en un plan nacional de salud). Esto puede ser pensado de esta manera, pues en el caso de la salud no existe un mercado que determine estas cuestiones al mismo tiempo, equilibre la disponibilidad a pagar de las personas con los costos marginales de los servicios y otorgue, entonces, señales a quienes quieren invertir, a los oferentes y a los consumidores. Lo que se encuentra en realidad es que los recursos financieros corrientes y las decisiones de inversión, fruto de la planificación, deben confluir a través de sistemas diferentes al mercado, esto es, a través de la planeación y de esquemas de asignación específicos. De este modo, el sistema de pagos, como esquema de asignación de recursos, de ninguna manera puede reemplazar la acción de la planificación de salud, si no que es su complemento y debe estar al servicio y orientado a los objetivos de la integración de los servicios.

El financiamiento de una RISS debe basarse en criterios de eficiencia y equidad asociados a la repartición adecuada del riesgo $^{4}$ entre financiadores y proveedores, de modo que logre balancear costos y calidad y se concrete en un presupuesto que

\footnotetext{
${ }^{4}$ Se trata de un riesgo financiero asociado a la pérdida financiera por efecto de la enfermedad y a la variabilidad de la utilización y de los costos, debido a la heterogeneidad de estados de salud de la población, y dados ciertos niveles de acceso.
} 
CUADRO 2. Tipos de sistemas o mecanismos de pago a proveedores según sus incentivos básicos

\begin{tabular}{lll}
$\begin{array}{c}\text { Tipo de sistema o } \\
\text { mecanismo de pago }\end{array}$ & \multicolumn{1}{c}{ Característica central del Incentivo } & \multicolumn{1}{c}{ Ejemplos } \\
\hline Pagos prospectivos & $\begin{array}{l}\text { Incentivos a la eficiencia, pero también a la selección/ } \\
\text { exclusión de prestaciones o personas }\end{array}$ & $\begin{array}{l}\text { Pagos de servicios agrupados a centros de salud predeterminados por } \\
\text { convenios o contratos } \\
\text { Per cápita al primer nivel de atención predeterminado por convenios o contratos }\end{array}$ \\
Pagos retrospectivos & $\begin{array}{l}\text { Los proveedores eligen número, tipo y calidad de los } \\
\text { servicios sin ocuparse de las consecuencias financieras }\end{array}$ & $\begin{array}{l}\text { Pago por acto para reembolso de servicios prestados por centros de salud } \\
\text { Pago per diem en hospitales por unidades día-pacientes }\end{array}$ \\
Pagos mixtos & $\begin{array}{l}\text { Pueden balancear los incentivos minimizando la selección } \\
\text { de personas o servicios y mejorando la eficiencia }\end{array}$ & $\begin{array}{l}\text { Grupos relacionados por el diagnóstico para pago a grupo de hospitales, } \\
\text { considerando actividad y costos fijos } \\
\text { Presupuesto poblacional/per cápita ajustado por riesgos para pago a RISS, } \\
\text { calculado considerando costos fijos y la actividad esperada }\end{array}$ \\
\hline
\end{tabular}

Fuente: Elaboración propia y con base en 28.

RISS, redes integradas de servicios de salud.

logre capturar la variación de los costos, de los niveles de utilización y de salud, de modo controlar la heterogeneidad de la población a cargo, fomentando de ese modo mayor eficiencia.

Un sistema de pagos, en este caso, procura que el dinero siga al costo esperado de la necesidad sanitaria de las personas y comunidades hasta allí donde lo necesitan, promueve el principio de equidad en el acceso a los servicios (y su financiamiento) para una misma necesidad, (25) y permite la prestación de los servicios con eficiencia. En este sentido, la presupuestación basada en una capitación ajustada por las necesidades de la población fomenta la articulación entre los niveles de atención en la medida que constituye los recursos por persona que están disponibles para el conjunto de la organización y para todos sus niveles, y ayuda a generar la necesidad de coordinación adecuada de las redes de salud. Así, el financiamiento basado en un presupuesto poblacional, con un per cápita ajustado por las necesidades o riesgos de salud de las poblaciones, constituye una de las fórmulas más eficaces para fomentar la integración de los proveedores. (26)

La capitación ajustada por riesgo es un sistema de pago mixto con un aspecto prospectivo reflejado en el costo de la morbilidad esperada y la actividad, y otro retrospectivo basado en las estructuras o costos fijos que deben cubrirse; reconoce, de esta forma, la acción imprescindible de la planificación sanitaria en dichas estructuras.

Los sistemas mixtos de pago no son una mera colección de mecanismos de pago diferentes; consideran los dos aspectos mencionados -prospectivo y retrospectivo- para reemplazar el fracaso de los mecanismos de mercado en la asignación de recursos en salud mediante un mecanismo que reconozca las necesidades de incorporar la planificación en las decisiones de este tipo. De esta forma, un sistema de pago mixto supera el problema de enfatizar lo retrospectivo, pagando el gasto producido sin ningún proceso que fomente la eficiencia, así como lo prospectivo, que fomenta la exclusión de pacientes o prestaciones. Así, balancea la contradicción entre eficiencia y selección que, desde el punto de vista económico, caracteriza al mundo del financiamiento de la provisión de la salud. $(27,28)$ (Cuadro 2)

Si se presupuesta atención sanitaria a una población estimando sus consumos esperados, se está fomentando la búsqueda de la eficiencia en los proveedores a través de una correcta articulación entre los niveles de atención. Se fomenta el seguimiento de personas en el conjunto de problemas de salud que le afectarán durante la vida, el equilibrio del riesgo entre pagadores y proveedores y se estimula la capacidad resolutiva. (25) La cápita ajustada por riesgos generará incentivos a la contención de costos y a tener en cuenta las diferencias en la necesidad de las distintas poblaciones (28), contribuyendo a la equidad en mayor grado que otros esquemas de financiamiento. En efecto, cuando la distribución de recursos a los proveedores no está vinculada con las necesidades de la población se generan desigualdades de manera anticipada que bloquean el alcance de la igualdad en el acceso frente a igual necesidad (por ejemplo, al estimar el presupuesto sólo en base a la oferta sin tomar en cuenta las necesidades y la demanda). Esto implica la necesidad de la RISS de detectar la demanda no expresada e insatesfecha a través de intervenciones de su primer nivel de atención.

La presión de las tendencias demográficas (envejecimiento), de morbilidad (predominio de las enfermedades crónicas) y del cambio tecnológico sobre los costos es más manejable en un contexto donde se propicia una relación fluida entre los niveles de atención y pueden reasignarse recursos entre ellos. Así, cuando un proveedor de tipo RISS es responsable de la atención de salud de una población determinada le será imperioso que la atención ocurra en el nivel de asistencia que ofrezca mayor capacidad resolutiva para un determinado problema de salud, y podrá potenciar al primer nivel de atención para que cumpla ese rol. La capacidad resolutiva se entiende entonces, en términos económicos, como una relación entre los mejoramientos en los desenlaces de los problemas de salud y sus costos, y es una expresión clave de la eficiencia técnica del proveedor. (26)

Como se ha señalado, el pago a RISS ha sido menos tratado que otros, pero se pueden identificar diferentes experiencias desde hace bastante tiempo (29). En Australia, Brasil, Chile, Inglaterra, España (algunas Comunidades Autónomas), Italia, Canadá (provincia de Alberta), Dinamarca y Finlandia se utilizan presupuestos poblacionales a través de la capitación. En EE. UU., en los ambientes de las HMO como Kaiser y a partir de la implementación de las ACO, el presupuesto poblacional ajustado por riesgos es el elemento central de los incentivos en el financiamiento (Cuadro 3). Esto no significa que, en estas experiencias, no existan otros mecanismos de financiamiento en la misma red, pero idealmente ellos se combinan y la sumatoria de las cápitas resulta ser el techo presupuestario que contiene a todos los demás y en conjunto se encuentran alineados. ${ }^{5}$ Por

\footnotetext{
${ }^{5}$ Con el término "alineados" nos referimos a que el conjunto de mecanismos de pago de un sistema de pago presente incentivos coherentes con el logro de las metas de la red y que no sean contradictorios, es decir, que promuevan efectos en la misma dirección entre las organizaciones y agentes de la RISS.
} 
CUADRO 3. Pagos poblacionales per cápita a proveedores de salud en 10 países escogidos

\begin{tabular}{|c|c|c|c|}
\hline País & Sistema de salud o subsistema donde opera & $\begin{array}{l}\text { Áreas sujetas a la } \\
\text { asignación poblacional }\end{array}$ & $\begin{array}{l}\text { Tipo de ajustadores de riesgo usados } \\
\text { para corrección de la cápita }\end{array}$ \\
\hline Australia & Sistema público universal: Medicare & 17 áreas de salud & Demográficos, socioeconómicos, salud, geográficos \\
\hline Brasil & Sistema público universal: Sistema único de salud & 5564 municipios & No considera ${ }^{b}$ \\
\hline Chile & $\begin{array}{l}\text { Sistema público: Sistema nacional de seguridad social } \\
\text { (subsistema) }\end{array}$ & 320 municipios & Demográficos, socioeconómicos, geográficos ${ }^{b}$ \\
\hline Dinamarca & Sistema público universal: Sistema nacional de salud & 4 regiones de salud & Demográficos, socioeconómicos \\
\hline España (Cataluña) & Sistema público universal: Sistema nacional de salud & 5 áreas de salud & Demográficos, salud ${ }^{\mathrm{b}}$ \\
\hline Finlandia & Sistema público universal: Sistema nacional de salud & 452 municipios & Demográficos, salud, geográficos \\
\hline Inglaterra & Sistema público universal: Sistema nacional de salud & 211 CCG & Demográficos, salud, socioeconómicos ${ }^{b}$ \\
\hline Italia & Sistema público universal: Sistema nacional de salud & 21 regiones & Demográficos, salud ${ }^{b, c}$ \\
\hline
\end{tabular}

el contrario en muchos casos es posible encontrar, por ejemplo, pagos dirigidos a hospitales a través de grupos relacionados por el diagnóstico (GRD) dentro de organizaciones de salud integradas. También se pueden apreciar componentes asociados a resultados en la propia cápita poblacional o unas destinadas a los médicos o instituciones del primer nivel de atención.

El ajuste por riesgo de la cápita es necesario para dar cuenta de la heterogeneidad de los costos en salud, de modo de poder estimar lo mejor posible los costos esperados de la población a cargo. La técnica que cuenta con un amplio desarrollo considera el uso de información de la población sobre determinantes sociales de salud, factores demográficos o la morbilidad esperada para hacer los ajustes, (14) incluso la necesidad de llevar a cabo ajustes por factores que promuevan bienes públicos asociados a la oferta como la educación médica, dar servicios en áreas desatendidas o de difícil acceso, o la consideración de la promoción de la salud, entre otros. (23)

Un sistema de pago a RISS como el expuesto puede asociarse a lo que la literatura ha descrito como compra estratégica, que en términos normativos es un sistema de pago mixto que promueve un énfasis en el desempeño y una actitud activa en la distribución de los recursos y pagos, más que una meramente pasiva, pero al mismo tiempo asociada directamente con las necesidades de la población (31).

Para obordar este tipo de políticas se debe considerar que en la medida que se conocen los costos, la morbilidad, las condiciones de acceso de la población y los servicios que se debe entregar se puede hacer una mejor gestión en las redes. Se trata de medir y evaluar para gestionar, y la medida de la morbilidad poblacional es un punto de partida básico. (32) En los países de América Latina y el Caribe este avance ha sido muy lento; sin embargo, estos requisitos para la buena gestión y gobernanza de las redes de salud son, a su vez, necesarios para el desarrollo de sistemas de pago efectivos y acelerar su implementación parece un reto ineludible.

Finalmente, la experiencia muestra que estos no son procesos fáciles y que resulta complejo implementar mecanismos integrados y mas estretégicos $(33,34)$. La implementación de este tipo de mecanismos demanda desafíos institucionales y técnicos importantes, desde el desarrollo de capacidades hasta el fortalecimiento de la rectoría de los sistemas de salud para mantener y desarrollar estos sistemas y hacerlos creíbles y, por lo tanto, efectivos. (5) De ello también se deriva una adecuación de los presupuestos públicos para que reflejen la estructura de la RISS y puedan entonces ser útiles en la acción de la planificación en salud que debe recoger el sistema de pagos.

\section{CONCLUSIONES}

El aumento de los recursos públicos para salud que la gran mayoría de los países de la Región necesita debería destinarse a financiar un modelo de atención eficiente que promueva el acceso equitativo a la salud. Para avanzar en los objetivos y metas de salud se necesita adecuar al sistema de salud. Se ha demostrado que un sistema de salud cumple mejor su misión cuando se organiza como RISS basadas en atención primaria de la salud. Sin embargo, en términos relativos, hasta ahora ha existido menos atención al desarrollo del financiamiento en este contexto, y en general los sistemas de pago e incentivos se limitan a tratar a los proveedores, o a los mecanismos propiamente, de forma aislada.

Existe evidencia y experiencias que muestran que la mejor forma de financiar a los proveedores de salud es aquella que entienda que los sistemas deben ser mixtos, reconozca el rol de la planificación, el necesario incentivo a la actividad, acompañe la integración y potencie las posibilidades de coordinación, a fin de reemplazar la visión parcial y el énfasis equivocado en la competencia por uno de integración y colaboración, que sin embargo mantenga los incentivos a la eficiencia. En ese contexto, un mecanismo del tipo presupuesto poblacional con un per cápita ajustado por riesgos que determine un techo financiero para la RISS como elemento central del sistema de pagos es una herramienta efectiva para el logro de estos objetivos. Dentro de la RISS pueden coexistir otros métodos dirigidos a areas específicas de manera alineada con la asignación poblacional. 
La Región ha avanzado de manera lenta en este tipo de aplicaciones. Esto puede ser visto como una oportunidad y un desafío. La oportunidad es que se cuenta con experiencia acumulada no despreciable que es posible aprovechar; el desafío, que los cambios en este ámbito deben ser apoyados por la decisión política y los mecanismos institucionales respectivos, así como estar insertos de manera prioritaria en los procesos de transformación de los sistemas de salud hacia la salud universal que actualmente emprenden los países.

\section{Conflicto de intereses. Ninguno declarado.}

Declaración. Las opiniones expresadas en este manuscrito son responsabilidad del autor y no reflejan necesariamente los criterios ni la política de la RPSP/PAJPH o de la OPS.

\section{REFERENCIAS}

1. Organización Panamericana de la Salud. Resolución CD 53 R14. Estrategia para el acceso universal a la salud y la cobertura universal de salud. 54 Consejo Directivo, 66 sesión del comité regional de la OMS para las Américas. Washington D.C.: OPS; 2014.

2. Organización Panamericana de la Salud. Redes Integradas de Servicios de Salud: Conceptos, Opciones de Política y Hoja de Ruta para su Implementación en las Américas. Serie La Renovación de la Atención Primaria de Salud en las Américas, N. 4. Washington D.C.: OPS; 2010.

3. Shortell SM, Gillies RR, Anderson DA, Erickson KM, Mitchell JB. Remaking health care in America. Hosp Health Netw. 1996;70(6): 43-44.

4. Cid C, Riesco X. Impacto de la selección de los mecanismos de pago en la producción de atención de salud: ¿Están en línea con los objetivos sanitarios? Cuad méd-soc. (Santiago de Chile) 2008;48(1): 13-23.

5. Matahuer I, Dale E, Jowett M, Kutzin J. Purchasing of health services for universal health coverage: How to make it more strategic? Health Financing Policy Brief No. 6. Geneva: World Health Organization; 2019.

6. European Observatory on Health Systems and Policies/World Health Organization. Purchasing to improve health systems performance. Figueras J, Robinson R, Jakubowski E. Eds. New York: McGraw Hill; 2005.

7. The World Bank. Public Ends, Private Means: Strategic Purchasing of Health Services. Alexander S. Preker, Xingzhu Liu and Edit V. Velenyi. Editors. Washington D.C.: The World Bank; 2007. https:// elibrary.worldbank.org/doi/pdf/10.1596/978-0-8213-6547-2

8. The World Bank. Designing and Implementing Health Care Provider Payment Systems How-To Manuals. John C. Langenbrunner, Cheryl Cashin, Sheila O'Dougherty, Editors. Washington D.C.: The World Bank; 2009. https://openknowledge.worldbank.org/bitstream/handle/10986/13806/48599.pdf?sequence $=1$

9. European Observatory on Health Systems and Policies/World Health Organization. Diagnosis Related Groups in Europe. Reinhard Busse, Alexander Geissler, Wilm Quentin, Miriam Wiley, Editors. New York: McGraw Hill; 2011. http://www.euro.who.int/_data/ assets/pdf_file/0004/162265/e96538.pdf

10. European Observatory on Health Systems and Policies/World Health Organization. Paying for Performance in Health Care Implications for health system performance and accountability. Cheryl Cashin, Y- Ling Chi, Peter C. Smith, Michael Borowitz and Sarah Thomson, Eds. New York: McGraw Hill; 2014. http://www.euro. who.int/__data/assets/pdf_file/0020/271073/Paying-for-Performance-in-Health-Care.pdf

11. World Health Organization. Regional Office for the Western Pacific. Case-based payment systems for hospital funding in Asia: an investigation of current status and future directions. Manila: WHO Regional Office for the Western Pacific; 2015. https://apps.who. int/iris/handle/10665/208246

12. OECD. Better Ways to Pay for Health Care, OECD Health Policy Studies. París: OECD Publishing; 2017. https://doi. org/10.1787/9789264258211-en.

13. Ibern-Regas P. Integración asistencial: fundamentos y vías de avance. Colección Economía de la Salud y Gestión Sanitaria. Barcelona: Masson; 2006.
14. Cid C, Ellis RP, Vargas V, Wasem J, Prieto L. Global Risk-Adjusted Payments Models. World Scientific Handbook of Global Health Economics and Public Policy. 2016; 3(1):311-362.

15. Castro R, Cid C, Román A, Butala N. Changing Health Care Provider Incentives to Promote Prevention: The Chilean Case. Harvard Health Policy Rev. 2006;7(2):102-112.

16. Organización Panamericana de la Salud. Financiamiento de la salud en las Américas.Salud en las Américas 2017.https://www.paho.org/ salud-en-las-americas-2017 / ?post_type=post_t_es\&p=290\&lang=es

17. Organización Panamericana de la Salud. Salud Universal en el Siglo XXI: 40 años de Alma-Ata. Informe de la Comisión de Alto Nivel, 2019. Washington D.C.: OPS; 2019.

18. Villacrés T, Mena AC. Mecanismos de pago y gestión de recursos financieros para la consolidación del Sistema de Salud de Ecuador. Rev Panam Salud Publica. 2017;41:e51.

19. Ministerio de Salud. Asistencia Técnica al Ministerio de Salud para el diseño, articulación y desarrollo de los pilotos de aseguramiento universal en salud: sistematización de las experiencias registradas y apoyo en el ajuste y monitoreo del plan de implementación; 2010.

20. Vásquez ML, Vargas I, Nuño R, Toro N. Organizaciones sanitarias integradas y otros ejemplos de colaboración entre proveedores. Informe SESPAS 2012. Gaceta Sanitaria; 2012.

21. Ortún V, López-Casasnovas G, Puig J, Sabés R. El sistema de financiación capitativo: posibilidades y limitaciones. Fulls Economics del Sistema Sanitari. 2001;35:8-16.

22. Shortell SM, Anderson DA, Gillies RR, Mitchel JB, Morgan KL. Building Integrated Systems: The Holographic Organization. Healthcare Forum Journal. 1993;36(2):20-26.

23. Scheffler R, Bowers L, Fulton B, Connors C, Shortell S, Morrison I. A New Vision for California's Health Care System: Integrated Care Aligned Financial Incentives. Berkeley Forum, University of California Berkeley School of Public Health; 2013.

24. Whaley C, French III H, Scheffler R. Accountable Care Organizations in California: Market Forces at Work? Journal of Health Politics, Policy and Law. 2015;40 (4):689-703.

25. Ortún-Rubio V, López-Casasnovas G. Financianción capitativa: articulación entre niveles asistenciales y descentralización de las organizaciones sanitarias. Centre de Recerca en Economia i SalutCRES. Universidad Pompeu Fabra/Fundación BBVA. Documento de trabajo Nro. 3; 2014.

26. Cid C. Revisión de literatura y sistematización de información disponible sobre la asignación de recursos a nivel comunal. Santiago: Ministerio de Salud de Chile; 2012. http:/ / desal.minsal.cl/wp-content/uploads/2013/09/REVISION-DE-LITERATURA.pdf.

27. Newhouse JP. Reimbursing health plans and health providers: Selection versus efficiency in production. Journal of Economics Literature. 1996;34:1236-1263

28. Ellis R, Martins B, McKinnon Miller M. Provider Payment Method and Incentives. In Heggenhougen HK and Quah SR (eds.). International Encyclopedia of Public Health ( $2^{\text {nd }}$ Ed.); 2015.

29. Rice N, Smith P. Approaches to Capitation and Risk Adjustment in Health Care: An International Survey. Centre for Health Economics, University of York, October 1999.

30. Checkland K, McDermott I, Coleman A, et al. Complexity in the new NHS: longitudinal case studies of CCGs in England. BMJ Open 2016;6:e10199. doi:10.1136/bmjopen-2015- 010199 
31. Mathauer I, Dale E, Meessen B. Strategic purchasing for Universal Health Coverage: key policy issues and questions. A summary from expert and practitioners' discussions Geneva: World Health Organization; 2017.

32. Carreras M, Sánchez-Pérez I, Ibern P, Cordech J, Inoriza JM. Analysing the Costs of Integrated Care: A case on Model Selection of Chronic Care Purposes. Int J Integr Care. 2016;(3): 10:1-11. doi: http://dx.doi.org/10.5334/ijic.2422 17

33. Klasa K, Greer S, van Ginneken E. Strategic Purchasing in Practice: Comparing Ten European Countries. Health Policy. 2018;122:457-472.
34. Cashin C, Eicher R, Hartel L. Unleashing the potential of strategic purchasing. Health Finance and Governance Project. Bethesda: USAID; 2018.

Manuscrito recibido el 7 de mayo de 2020. Aceptado para su publicación, tras revisión, el 2 de julio de 2020.

\section{Financing of integrated health service networks}

\section{ABSTRACT}

Keywords
The countries of Latin America and the Caribbean need to increase their public resources in health to expand equitable and efficient access to health. The increase should finance a specific model with proven effectiveness, such as integrated health service networks (IHSN) based on primary health care. The global literature has not paid sufficient attention to financing IHSN; rather, it has focused on isolated facilities and agents, as well as on specific mechanisms. However, in the Region of the Americas, their development has been a necessity for years. An IHSN is a group of health organizations that offers coordinated health interventions and services to a population under their charge and assumes health and economic responsibility for achieving better health outcomes. A system of payment to an IHSN should be aimed at promoting the integrality of care and encouraging a focus on the life cycle of individuals, the articulation and the coordination of services. The risk-adjusted population budget is a possible and powerful mechanism to support the achievement of the objectives. Its development requires the recognition that the type of financing alone will not respond to the challenges and that there is a need for both health planning and health management. The technical, political and institutional challenges need to be addressed to succeed in this effort, which in turn must be embedded in the overall process of transforming health systems towards universal health.

Health care rationing; capitation fee; health systems; healthcare financing; Americas. 